\title{
Mentoria entre pares na escola médica: uma estratégia colaborativa durante a pandemia da Covid-19
}

\section{Peer mentoring in a medical school: a collaborative strategy during the Covid-19 pandemic}

\author{
Paulo Matheus Dorneles Martins ${ }^{1}$ (D) $\mid$ paulomdmartins@gmail.com \\ Vitória Xausa Bosak' (D) vitoria.bosak@gmail.com \\ Débora Lana De Césaro Oliveski ${ }^{1}$ (D) deboraoliveski@gmail.com \\ Bibiana Barcellos da Rosa Hoff' (1) bibianabarcellos28@gmail.com

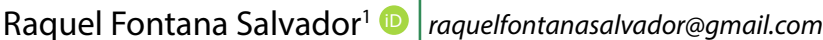 \\ João Ricardo Hass Massena' (D) joaoricardohm@gmail.com
}

\begin{abstract}
RESUMO
Introdução: Devido ao distanciamento social necessário em um contexto de pandemia, a educação médica passou por rápidas e profundas transformações, como a suspensão das atividades presenciais e a virtualização do ensino, exigindo imensa capacidade de adaptação dos novos ingressantes no curso de Medicina. Nesse cenário, foi implementado o projeto de extensão Aluno Mentor em Tempos de Pandemia, visando a uma melhor integração acadêmica e social dos calouros.
\end{abstract}

Relato de experiência: No projeto, um aluno veterano com maior experiência (aluno mentor) acolheu um aluno calouro (aluno mentorado), apoiando, orientando e guiando nas situações e dificuldades que surgiram ao longo do semestre. Além das reuniões de mentoria entre os alunos, foram realizados encontros de formação e supervisão com os alunos mentores.

Discussão: A mentoria pode colaborar de maneira substancial no processo de acolhimento, acompanhamento e estabelecimento de vínculos dos estudantes na fase inicial da sua vida universitária, promovendo o seu desenvolvimento acadêmico e pessoal. Ao mesmo tempo que a mentoria entre iguais apoia o calouro, ela é uma oportunidade para potencializar a formação pessoal, acadêmica e profissional do aluno mentor, possibilitando o desenvolvimento de habilidades sociais, de comunicação, orientação, organização e liderança dos alunos veteranos.

Conclusão: A implementação do projeto de mentoria entre pares constitui uma potente estratégia de apoio aos estudantes de Medicina, de modo a facilitar a integração deles ao curso.

Palavras-chave: Pandemias; Empatia; Acolhimento; Tutoria; Faculdades de Medicina.

\begin{abstract}
Introduction: Due to the social distancing needed during the current pandemic, medical school has had to undergo deep and rapid transformation, such as the suspension of classroom activities and the virtualization of teaching, demanding high levels of adaptation skills mainly of the new medical students. In this scenario, Project 'Peer Mentor in the Pandemic' was implemented, aiming to achieve better results either from a social or academic perspective for these new students.
\end{abstract}

Experience Report: In the project a senior/experienced student ('Peer Mentor') is in charge to receive a new student ('Student Mentee'), providing support, orientation and applicable guidance to help them through difficulties and issues that arise during the semester. Besides the peer mentoring meetings, training and supervision sessions were held for the Peer Mentors.

Discussion: The mentorship could substantially improve the integration, continuous development and bonding process of the students at the start of their medical training, promoting positive impacts on a social and academic level. Similarly, peer mentorship also enhances the senior student's skills, such as social skills, communication skills, guidance principles, organizational abilities and leadership.

Conclusion: Implementing peer mentoring may represent a strong strategy to support medical students, providing them with an easier route to being assimilated into this new environment.

Keywords: Pandemic; Empathy; User Embracement; Mentoring; Medical School.

1 Universidade do Vale do Rio dos Sinos, São Leopoldo, Rio Grande do Sul, Brasil.

Editora: Patrícia Lacerda Bellodi.

Recebido em 02/03/21; Aceito em 07/04/21.

Avaliado pelo processo de double blind review. 


\section{INTRODUÇÃO}

A pandemia da coronavirus disease 2019 (Covid-19) impactou profundamente as instituições de ensino superior e vem apresentando inúmeros desafios para as escolas de Medicina. O distanciamento social e a virtualização do ensino tornam ainda mais complexo o processo de transição do ensino médio para o ensino superior. Além disso, já é sabido sobre a alta incidência de transtornos mentais entre os estudantes de Medicina, com a redução da qualidade de vida e a alta incidência de quadros de ansiedade e depressão agora agravada pela pandemia ${ }^{1,2}$. Há, dessa forma, o risco de um processo de naturalização da depreciação da saúde mental por parte dos estudantes de Medicina, fato que pode interferir negativamente na busca por ajuda ${ }^{3}$.

Nessa perspectiva, surge a necessidade de construirmos estratégias que facilitem uma melhor integração acadêmica e social dos calouros. Assim, insere-se o projeto de mentoria entre pares: uma relação horizontal que visa contribuir para o ingresso ao ambiente universitário e apoiar as demandas surgidas ao longo dessa etapa.

Na universidade, já é desenvolvida a tutoria integradora, que constitui um componente curricular obrigatório do curso de Medicina, em que é designado um professor para acompanhar um pequeno grupo de estudantes desde o primeiro semestre até a conclusão da graduação. Nessa disciplina, abordam-se os seguintes temas: gestão do próprio conhecimento, desempenho acadêmico, atitudes, formação médica e carreira, processos de aprendizagem, identificação de problemas e conflitos, assim como ampliação do olhar sobre as dimensões cognitivas e afetivas do ser médico. A tutoria é realizada de maneira semanal ou quinzenal (em dependência do semestre) e conta com o registro dessas atividades em portfólio e a avaliação pelo professor por meio de conceito.

Já a proposta de mentoria entre pares surge como um projeto de extensão, contando com a participação e adesão voluntária dos alunos mentores e alunos mentorados. Ela é destinada exclusivamente aos alunos calouros, com o objetivo principal de integrá-los ao curso e brindar o suporte necessário nessa etapa. O projeto não prevê hierarquização e esquemas rígidos, mas sim o estabelecimento de uma relação horizontal, possibilitando a formação de percursos singulares, a partir da escuta ativa das demandas trazidas pelos alunos mentorados 4 . Com a mentoria entre pares os calouros podem se sentir mais à vontade em expressar suas inseguranças, dúvidas e insatisfações, ao mesmo tempo que os alunos mentores conseguem compreender melhor essas demandas.

A mentoria é então uma relação em que uma pessoa mais experiente oferece apoio e orientação a um outro indivíduo no começo de sua jornada, com intervenção de suporte a esse momento de transição, ajudando na afiliação dos novos alunos ao universo do ensino superior e ao curso de Medicina ${ }^{5}$. Portanto, ambas as estratégias (tutoria integradora e mentoria) não são antagônicas, mas podem atuar de maneira sinérgica, complementando-se e potencializando o processo de formação dos futuros médicos.

Por meio de uma interação construída na confiança, os programas de mentoria têm o potencial de contribuir para a construção de um espaço seguro de partilha de sentimentos, angústias e preocupações ${ }^{6}$. Nesse processo de acompanhar o aluno na fase inicial de sua vida universitária, pretende-se facilitar o seu desenvolvimento humano e discente: a mentoria entre pares tem ganhado força ao facilitar a abordagem de assuntos considerados de grande importância pelos alunos, como o desempenho acadêmico, a saúde mental e o bem-estar ${ }^{7,8}$.

Cada vez mais, destaca-se a importância dos programas de mentoria na formação integral do estudante de Medicina, na medida em que valorizam e reconhecem os componentes afetivo, ético, social e cognitivo do processo educacional ${ }^{9}$.

Nesse contexto, este relato traz a experiência do projeto de extensão Aluno Mentor em Tempos de Pandemia, desenvolvido no curso de Medicina da Universidade do Vale do Rio dos Sinos (Unisinos).

\section{RELATO DA EXPERIÊNCIA}

O projeto foi desenvolvido com alunos de Medicina da Unisinos, no segundo semestre de 2020, momento em que as atividades acadêmicas presenciais foram suspensas e todo o primeiro semestre do curso foi desenvolvido de maneira remota devido à pandemia da Covid-19. O desenvolvimento e a execução do projeto contaram com o apoio do Núcleo de Apoio Pedagógico (NAP), colegiado responsável pela assessoria e apoio aos processos formativos. O NAP participa efetivamente no delineamento teórico e na elaboração do projeto, na seleção dos alunos mentores, no planejamento das atividades de mentoria e do programa de formação dos alunos mentores, e no processo de avaliação.

Os alunos mentores foram selecionados por meio da indicação dos professores tutores, sendo considerados os alunos matriculados a partir do terceiro semestre com disponibilidade de duas horas semanais (totalizando 30 horas semestrais). O aluno selecionado deveria reunir uma série de características e habilidades preconizadas para a condução da mentoria, como perfil acolhedor, empático e colaborativo.

O grupo de alunos mentores era constituído por 13 discentes matriculados do terceiro ao sétimo semestre. Já o grupo de alunos mentorados era constituído por 34 discentes que haviam ingressado recentemente na graduação de Medicina, em sua maioria jovens adultos do sexo feminino e 
provenientes da região metropolitana de Porto Alegre.

Cada aluno mentor, a partir da orientação do professor supervisor, acompanhou de dois a três alunos mentorados, visando cumprir os seguintes objetivos:

- Acolher, acompanhar e estabelecer vínculos com o aluno na fase inicial de sua vida universitária, facilitando o seu desenvolvimento acadêmico e pessoal.

- Propiciar uma melhor integração do calouro ao curso, apresentando recursos, espaços e pessoas.

- Apoiar na resolução de problemas que possam ocorrer durante o processo de adaptação ao novo contexto.

- Potencializar a formação pessoal, acadêmica e profissional dos alunos mentores, possibilitando que desenvolvam habilidades sociais, de comunicação, orientação, organização e liderança.

- Promover interação para socialização, autorregulação e adaptação dos estudantes.

Os alunos mentores participaram de reuniões de planejamento e alinhamento com a coordenação do projeto e da aula inaugural do semestre, em que tiveram o primeiro contato com os seus alunos mentorados. Já os calouros participaram de uma reunião com a coordenação do curso e do projeto para apresentação da proposta de mentoria. Na primeira semana de aula, foi enviado a cada aluno mentorado um questionário de primeiras impressões, por meio do Google Forms. Nesse questionário, os discentes registraram as expectativas em relação ao curso e à universidade, a percepção sobre o processo de acolhimento e a inserção no ambiente virtual, assim como indicaram dúvidas, eventuais dificuldades e interesses.

O projeto contemplou mentorias individuais e em grupo, reuniões de supervisão e encontros de formação. Cabe ressaltar que todas as atividades foram desenvolvidas na modalidade on-line. Durante o período de exames acadêmicos, realizou-se uma atividade integradora que reuniu os alunos mentores e os mentorados, na qual se desenvolveu um trabalho em grupos com metodologias ativas e mindfulness.

O projeto contava com uma coordenação e um conjunto de professores colaboradores que contribuíram para a efetivação do programa de formação dos alunos mentores. Responsável pela gestão do projeto, a coordenação era composta por quatro professores do curso de Medicina que, em sua maioria, já desempenhavam outras funções em órgãos colegiados ou de gestão da universidade:

- Coordenadora pedagógica do curso de Medicina, integrante da equipe de formação docente e do NAP.
- Coordenadora do programa de aprendizagem dos três primeiros semestres e integrante do NAP.

- Coordenador do programa de tutorias integradoras.

- Professor de Medicina com especialização em Neurologia Pediátrica.

O professor de Neurologia Pediátrica era o coordenadorgeral do projeto e também exercia a supervisão dos alunos mentores por meio de reuniões em grupo e individuais, nas quais discutia dificuldades, planejava intervenções e desenvolvia potencialidades surgidas ao longo desse processo.

Visando qualificar a mentoria, foi realizado um programa de formação destinado aos alunos mentores. Durante o semestre, realizaram-se três encontros virtuais (totalizando 12 horas-aula), nos quais se utilizaram metodologias ativas e se abordaram os seguintes temas:

1) Escuta ativa, autoconhecimento e comunicação não violenta: tema desenvolvido por uma professora com formação em Jornalismo, mestrado em Antropologia das Organizações e especialista em Comunicação e Gestão Estratégica de Recursos Humanos, com área de atuação em relações interpessoais, autodesenvolvimento pessoal e profissional, comunicação não violenta, mindfulness e desenvolvimento do potencial humano.

2) Aprender a aprender: tema desenvolvido por uma professora de Neurologia, com mestrado em Ciências da Reabilitação e doutoranda em Neurociências, com ênfase em qualidade de vida e resiliência.

3) Saúde mental dos estudantes de Medicina: tema desenvolvido por uma professora de Psiquiatria, com doutorado na área e especialização em Psicoterapia.

\section{Desenvolvimento da mentoria}

Para o início das mentorias, havia uma recomendação da coordenação quanto a estrutura, calendário, periodicidade, temas e meios para a realização das atividades. Contudo, acima disso, preconizaram-se a escuta ativa e a individualização do processo. Houve, portanto, uma flexibilidade de formatos de acordo com as demandas trazidas pelos alunos mentorados e o desenvolvimento do fenômeno grupal entre alunos mentorados e aluno mentor.

As mentorias, usualmente, possuíam uma ocorrência semanal, com a participação do aluno mentor e do aluno mentorado. Durante os encontros, os alunos ingressantes 
Figura 1. Estrutura do projeto de extensão Aluno Mentor em Tempos de Pandemia.

\section{Projeto Aluno Mentor em tempos de Pandemia}

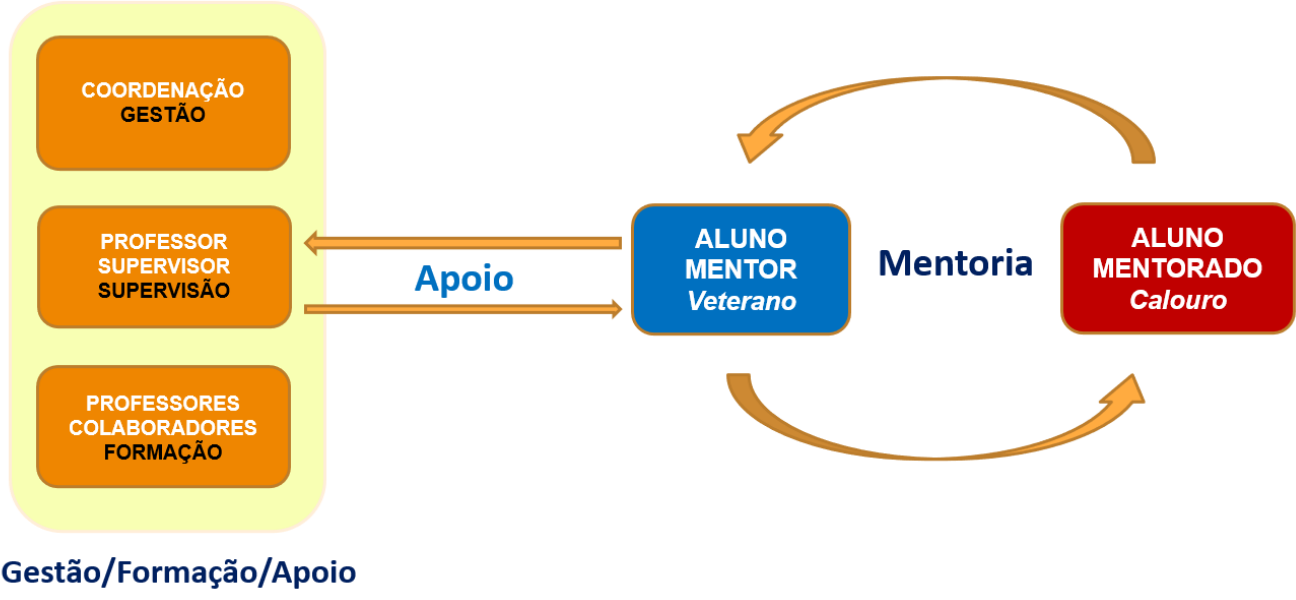

Fonte: Elaborada pelos autores.

não só esclareciam dúvidas gerais sobre o curso e os métodos de estudo, como também questionavam a respeito das experiências prévias dos alunos mentores na nova faculdade e compartilhavam as expectativas e angústias surgidas nessa etapa. A saúde mental foi uma demanda bastante frequente.

Os encontros da mentoria ocorriam, geralmente, via plataforma Microsoft Teams, que a instituição disponibiliza à comunidade universitária. Além da plataforma, foram muito utilizados aplicativos de mensagens instantâneas, o que possibilitou sanar dúvidas do cotidiano de maneira mais eficaz. Os temas abordados em cada mentoria giravam em torno das demandas trazidas pelos alunos, e as temáticas eram sugeridas pela coordenação do projeto, sendo as principais: conversa sobre a universidade e as primeiras impressões; apresentação de recursos, espaços e pessoas; dúvidas sobre as plataformas Moodle e Teams, as ligas acadêmicas, o acesso à biblioteca virtual e outros recursos; preparação para as provas; métodos de estudos; avaliações; técnicas de concentração e relaxamento; saúde mental; desempenho acadêmico; interesses pessoais e profissionais futuros; entre outros temas.

Os alunos mentores tinham reuniões periódicas com o professor supervisor com o objetivo de discutir e apoiar o processo de mentoria. Essas supervisões eram realizadas em grupo e de forma individual, de maneira periódica e extraordinária, quando demandadas pelo aluno mentor.

\section{Avaliação do projeto}

Ao final do semestre, foi realizada a avaliação do projeto pelos alunos mentores e alunos mentorados, por meio do preenchimento de questionário no Google Forms. A avaliação não identificava o aluno, e o objetivo era medir a satisfação dos participantes com o projeto, com respostas que partiam de uma escala de um até dez: quanto mais satisfeito, mais próximo de dez, e, quanto menos satisfeito, mais próximo de zero. Nesse mesmo questionário, foi aberto um espaço para que os participantes pudessem transmitir suas impressões, críticas e sugestões.

Quando indagados sobre a satisfação com o projeto, $62,5 \%$ dos alunos mentorados atribuíram nota nove; e $37,5 \%$, dez. Já entre os alunos mentores, 27,3\% indicaram nove para experiência; e 72,7\%, dez. Entre os alunos mentorados, $100 \%$ qualificaram com dez o acolhimento recebido e a disponibilidade dos alunos mentores para o esclarecimento de dúvidas sobre o curso e a universidade. De igual maneira, a totalidade dos alunos mentorados recomendou o projeto para os próximos calouros. Já entre os alunos mentores, $81,8 \%$ qualificaram com dez a satisfação com o programa de formação, e 63,6\% também deram pontuação máxima quando perguntados em que medida consideraram que foram cumpridos os objetivos previstos inicialmente para o projeto.

Cada aluno mentor também produziu uma memória escrita de forma reflexiva sobre as vivências do projeto. Nessas memórias, os alunos mentores relataram as suas expectativas iniciais e refletiram sobre suas experiências, elencando os principais desafios e potencialidades percebidos durante o exercício da mentoria. Também descreveram como foi o seu aprendizado e em que medida os encontros de formação contribuíram para o seu crescimento pessoal, acadêmico e profissional.

\section{DISCUSSÃO}

Durante a pandemia da Covid-19, os estudantes universitários podem ter forte impacto negativo em sua saúde 
mental, sendo comuns sintomas de ansiedade, medo e solidão, o que aumenta o risco de suicídio e abuso de substâncias. Somase a esse cenário de incerteza a preocupação dos discentes com a própria formação por meio de atividades a distância' ${ }^{2}$ O início de um curso como o de Medicina, desenvolvido em tempo integral e habitualmente caracterizado por ambientes em que muitas vezes há alta competitividade e individualismo, exige grande capacidade de adaptação e autorregulação.

É natural que, no processo de integração do calouro na universidade, surjam muitas dúvidas, incompreensões, tensões e situações de conflitos. A necessidade da construção de relações de troca e apoio suscitou importantes reflexões sobre o papel do aluno mentor nesse contexto. Para isso, recorremos ao triângulo do drama de Karpman, modelo social de interação que mapeia a relação entre indivíduos, para refletir e pautar a atuação dos alunos mentores, com uma atenção especial para que eles não reproduzissem o papel de salvadores, colocando o aluno mentorado no papel de vítima ${ }^{10}$. Ambos deveriam ser ativos nessa trajetória, e caberia ao aluno mentorado encontrar as respostas às suas questões a partir da própria reflexão, sem que elas fossem apontadas pelo aluno mentor. Afinal, antecipar as respostas não encurta o caminho, mas atrapalha o processo e gera dependência e não autonomia ${ }^{11}$.

Assim, foram cruciais as atividades de formação com os alunos mentores, nas quais refletiam sobre o papel deles nesse processo, de modo a qualificar a prática de mentoria. A discussão das inúmeras situações vivenciadas nesse processo possibilitou a ressignificação do trabalho que já estava em curso.

É importante ressaltar a complexidade que a mentoria assumiu ao ser também um espaço de cuidado com o outro, já que o ambiente universitário é tradicionalmente individualista e competitivo, focado no desempenho acadêmico. Isso se reflete na capacidade de cada mentoria em assumir percursos singulares a partir de uma escuta ativa e qualificada, ao mesmo tempo que traz o desafio da criação de vínculo e obtenção de engajamento.

Isso se traduznoaltograu de satisfação dosalunosmentores e mentorados com o projeto, já que houve o cumprimento dos objetivos propostos, o que gera benefício para ambos.

Porém, os benefícios da mentoria não se restringem apenas ao aluno mentor eao mentorado, uma vez que o currículo oculto e a qualidade das relações estabelecidas dentro do ambiente acadêmico também afetam o produto da formação: $o$ atendimento médico ${ }^{12,13}$. Afinal, uma das formas eficazes de aprendizado é por meio do exemplo e da experimentação. Assim, por meio do cuidado dentro das instituições, o futuro médico potencializa a sua própria capacidade de cuidar do paciente de forma mais qualificada e humana.

Nesse sentido, acredita-se que é possível promover e desenvolver a empatia por meio de práticas e treinamento de habilidades interpessoais e de comunicação, como os realizados para os alunos mentores ${ }^{14}$. Portanto, acreditamos que a mentoria pode impactar uma atuação médica de maior qualidade por meio da postura empática do profissional, que já foi associada com maior adesão do tratamento pelos pacientes, alta acurácia de diagnósticos, melhores desfechos clínicos, maior satisfação dos pacientes e maiores níveis de realização profissional do médico ${ }^{15}$.

\section{CONCLUSÃO}

Os resultados de exercitar a mentoria entre alunos calouros e alunos veteranos mostraram-se satisfatórios para ambos $^{12}$. Tornou-se uma intervenção eficaz que proporcionou o suporte necessário para alunos iniciantes nas escolas de Medicina. Além disso, possibilitou aos alunos mentores o desenvolvimento de habilidades de comunicação, solução de problemas e trabalho em equipe.

Evidencia-se também a dimensão psicossocial existente no decorrer do projeto, não sendo promovido e estimulado apenas o âmbito acadêmico. $O$ compartilhamento de vivências entre alunos de diferentes anos de formação possibilita a autorreflexão desses indivíduos sobre as suas próprias experiências ${ }^{16}$.

Todavia, apesar de seus notórios efeitos positivos, há uma recente revisão do tema que aborda a carência de estudos sobre a mentoria de pares na formação médica ${ }^{17}$. Nessa perspectiva, a mentoria entre pares, a partir das informações afirmativas no presente relato de experiência, deveria ser incentivada não apenas em contextos excepcionais como o que vivemos atualmente, mas também de maneira permanente $\mathrm{e}$ curricular, visando criar um ambiente mais inclusivo e saudável para todos os seus alunos.

\section{CONTRIBUIÇÃO DOS AUTORES}

Todos os autores participaram do delineamento teórico, da prática educacional e da redação do artigo, sob a orientação do professor João Ricardo Hass Massena.

\section{CONFLITO DE INTERESSES}

Declaramos não haver conflito de interesses.

\section{FINANCIAMENTO}

Declaramos não haver financiamento.

\section{REFERÊNCIAS}

1. Rodrigues BB, Cardoso RRJ, Peres CHR, Marques FF. Aprendendo com o imprevisível: saúde mental dos universitários e educação médica na pandemia de Covid-19. Rev Bras Educ Med. 2020;44(1):1-4. [acesso em 25 fev 2021]. Disponível em: https://www.scielo.br/scielo.php?pid=S0100 55022020000500302\&script=sci_arttext. 
2. Conceição LS, Batista CB, Dâmaso JGB, Pereira BS, Carniele LC, Pereira GSI. Saúde mental dos estudantes de medicina brasileiros: uma revisão sistemática da literatura. Avaliação: Revista da Avaliação da Educação Superior. 2019;24(3):785-802.

3. Leão PBOS, Martins LANER, Menezes PR, Bellodi PL. Bem-estar e busca de ajuda: um estudo exploratório entre alunos de Medicina ao final curso. Rev Assoc Med Bras. 2011;57(4):379-86.

4. Holliday M. Coaching, mentoring and managing: breakthrough strategies to solve performance problems and build winning teams. Franklin Lakes: Career Press; 2001

5. Bellodi PL. O Programa Tutores e a integração dos calouros na FMUSP. Rev Bras Educ Med. 2004;28(3): 204-14.

6. Ribeiro MMF, Martins AF, Fidelis GTA, Goulart GC, Molinari LC, Tavares EC. Tutoria em escola médica: avaliação por discentes após seu término e ao final do curso. Rev Bras Educ Med. 2013;37(4):509-14.

7. Andre C, Deerin J, Leykum L. Students helping students: vertical peer mentoring to enhance the medical school experience. BMC Res Notes. 2017;10(1):1-7 [acesso em 1 mar 2021]. Disponível em: https://www.ncbi. nlm.nih.gov/pmc/articles/PMC5414204/.

8. Singh S, Singh N, Dhaliwal U. Near-peer mentoring to complement faculty mentoring of first-year medical students in India. J Educ Eval Heal Prof. 2014;11(12):3-6. [acesso em 1 mar 2021]. Disponível em: https://pubmed. ncbi.nlm.nih.gov/24980428/

9. Moreia SNT, Albuquerque ICS, Pinto Jr FELP, Gomes AHB. Programa de Mentoria do curso de Medicina da Universidade Federal do Rio Grande do Norte: Atividades integrativas em foco. Rev Bras Educ Med. 2020;44(4):1-6.
10. Karpman S. Fairy tales and script drama analysis. Transactional Analysis Bulletin. 1968;7(26):39-43 [acesso em 3 mar 2021]. Disponível em: https:// www.karpmandramatriangle.com/pdf/DramaTriangle.pdf.

11. Xausa MR. Coaching e mentoring. Rio de Janeiro: FGV Management; 2017. $101 \mathrm{p}$.

12. Souza MG, Reato LFN, Bellodi PL. Ressignificando a relação entre calouros e veteranos: mentoria de pares na visão de alunos mentores. Rev Bras Educ Med. 2020;44(4):1-9.

13. Rojas AO. "Curriculum oculto" en medicina: una reflexión docente. Rev Med Chil. 2012;140(9):1213-7 [acesso em 1 mar 2021]. Disponível em: https:// scielo.conicyt.cl/scielo.php?pid=S0034-98872012000900017\&script=sci_ arttext\&tlng $=\mathrm{p}$.

14. Schweller M. O ensino de empatia no curso de graduação em medicina [tese]. Campinas: Universidade Estadual de Campinas; 2014

15. Fernández-Olano C, Montoya-Fernández J, Salinas-Sánchez AS Impact of clinical interview training on the empathy level of medical students and medical residents. Med Teach. 2008;30:322-4 [acesso em 4 mar 2021]. Disponível em: https://www.tandfonline.com/doi/ full/10.1080/01421590701802299?scroll=top\&needAccess=true.

16. Abdolalizadeh P, Pourhassan S, Gandomkar R, Heidari F, Sohrabpour AA Dual peer mentoring program: exploring the perceptions of mentors and mentees. Med J Islam Repub Iran. 2017;31(1):1-4.

17. Akinla $\mathrm{O}$, Hagan $\mathrm{P}$, Atiomo W. A systematic review of the literature describing the outcomes of near-peer mentoring programs for first year medical students. BMC Med Educ. 2018;18(98):1-8. 